\title{
New insights about phenotypic heterogeneity within Propionibacterium freudenreichii argue against its division into subspecies
}

\author{
Rosangela de Freitas ${ }^{1,2,3}$ - Marie-Noelle Madec ${ }^{2,3}$ - Victoria Chuat ${ }^{2,3}$. \\ Marie-Bernadette Maillard ${ }^{2,3}$ - María C. Abeijón Mukdsi ${ }^{2,3,4}$. \\ Hélène Falentin $^{2,3}$ - Antonio Fernandes de Carvalho ${ }^{1}$. \\ Florence Valence $^{2,3}$ - Anne Thierry ${ }^{2,3}$
}

Received: 17 October 2014 /Revised: 8 April 2015 / Accepted: 9 April 2015 / Published online: 8 May 2015

(C) The Authors 2015. This article is published with open access at Springerlink.com

\begin{abstract}
Propionibacterium freudenreichii is widely used in Swiss-type cheese manufacture, where it contributes to flavour and eye development. It is currently divided into two subspecies, according to the phenotype for lactose fermentation and nitrate reduction $\left(\mathrm{lac}^{+} /\right.$nit $^{-}$and $\mathrm{lac}^{-} /$nit $^{+}$for P. freudenreichii subsp. shermanii and subsp. freudenreichii, respectively). However, the existence of unclassifiable strains $\left(\mathrm{lac}^{+} / \mathrm{nit}^{+}\right.$and $\left.\mathrm{lac}^{-} / \mathrm{nit}^{-}\right)$has also been reported. The aim of this study was to revisit the relevance of the subdivision of $P$. freudenreichii into subspecies, by confirming the existence of unclassifiable strains. Relevant conditions to test the ability of $P$. freudenreichii for lactose fermentation and nitrate reduction were first determined, by using 10 sequenced strains, in which the presence or absence of the lactose and nitrate genomic islands were known. We also determined whether the subdivision based on lac/nit phenotype was related to other phenotypic properties of interest in cheese manufacture, in this case, the production of aroma compounds, analysed by gas chromatography-mass spectrometry, for a total of 28 strains. The results showed that a too short incubation time can lead to false negative for lactose fermentation and nitrate reduction. They confirmed the existence of four lac/nit phenotypes instead of the two expected, thus leading to 13 unclassifiable strains out of the
\end{abstract}

Anne Thierry

anne.thierry@rennes.inra.fr

1 Departamento de Tecnologia de Alimentos, Universidade Federal de Viçosa, Viçosa, MG, Brazil

2 INRA, UMR1253 Science et Technologie du Lait et de l'OEuf, 35042 Rennes, France

3 AGROCAMPUS OUEST, UMR1253 Science et Technologie du Lait et de l'OEuf, 35042 Rennes, France

4 Present address: Centro de Referencia para Lactobacilos (CERELA-CONICET), Chacabuco 145, (4000), Tucumán, Argentina 
28 characterized ( $7 \mathrm{lac}^{+} /$nit $^{+}$and $6 \mathrm{lac}^{-} /$nit $\left.^{-}\right)$. The production of the 15 aroma compounds detected in all cultures varied more within a lac/nit phenotype (up to 20 times) than between them. Taken together, these results demonstrate that the division of $P$. freudenreichii into two subspecies does not appear to be relevant.

Keywords Propionibacteria $\cdot$ Subspecies · Biodiversity $\cdot$ Volatile fingerprint $\cdot$ Flavour compound

\section{Introduction}

Propionibacterium freudenreichii is the main dairy propionibacteria species used as secondary culture in cheese manufacture, in particular for Swiss-type cheeses (Cummins and Johnson 1986). It produces varied aroma compound in cheese from lactate fermentation, amino acid catabolism, and milk fat hydrolysis, and $\mathrm{CO}_{2}$. These products significantly contribute to the development of the typical flavour and the formation of eyes in Swiss-type cheeses (Thierry et al. 2011, Abeijon Mukdsi et al. 2014). The production of aroma compounds is highly strain-dependent (Yee et al. 2014). Moreover, P. freudenreichii has potentials as bioprotective cultures by producing antimicrobial compounds such as bacteriocins and organic acids (Tharmaraj and Shah 2009) and antifungal peptides (Jan et al. 2007). It is also able to produce a variety of beneficial compounds for human health, such as vitamin B12 and folic acid (Hugenholtz et al. 2002), and some strains have been proposed as probiotics, due to their ability to modulate intestinal microbiota through their bifidogenic effect and to anti-inflammatory effects and antimutagenic properties, among others (Cousin et al. 2011).

P. freudenreichii is currently divided into two subspecies, on the basis of two phenotypical criteria (Cummins and Johnson 1986). P. freudenreichii subsp. freudenreichii does not ferment lactose and possess a nitrate reductase activity (lac ${ }^{-} /$ nit $^{+}$), whereas it is the opposite for $P$. freudenreichii subsp. shermanii $\left(\mathrm{lac}^{+} / \mathrm{nit}^{-}\right)$. However, the existence of strains harbouring the two other possible phenotypes $\left(\mathrm{lac}^{+} /\right.$ nit $^{+}$or lac ${ }^{-} /$nit $^{-}$) has occasionally been mentioned (de Carvalho et al. 1994; Kaspar 1982; Moore and Holdeman 1974; Vorobjeva 1999). More recently, in a study which characterized a collection of 113 strains of $P$. freudenreichii, more than $25 \%$ of strains could not be assigned to a subspecies, and were labelled "pheno+" $\left(\mathrm{lac}^{+} / \mathrm{nit}^{+}\right)$or "pheno" ${ }^{-"}\left(\mathrm{lac}^{-} / \mathrm{nit}^{-}\right)$(Dalmasso et al. 2011). The ability to ferment lactose and reduce nitrate results from the presence of genomic islands (Falentin et al. 2010; Loux et al. 2015). Regarding the use of $P$. freudenreichii for cheese ripening, the ability to ferment lactose can be an important criterion in some cheese varieties containing residual lactose at the beginning of the ripening, therefore resulting in the choice of strains of the subspecies $P$. freudenreichii subsp. shermanii. However, strains harbouring the phenotype $\mathrm{lac}^{+} / \mathrm{nit}^{+}$could be also chosen in this case. Besides, the possible relationships between the subdivision of the species and other technologically important properties are unknown.

Molecular methods have failed up to date to distinguish $P$. freudenreichii subspecies (Dasen et al. 1998; Fessler et al. 1999; Tilsala-Timisjarvi and Alatossava 2001), and therefore phenotypic methods are still required. Some discrepancies between studies 
could also have been due to a lack of standardization of phenotypical tests, since it is known that experimental conditions such as the presence of $\mathrm{O}_{2}$, temperature, and incubation time can influence the results observed (Busse et al. 1996; Swart et al. 1998).

The aim of this study was to revisit the relevance of the subdivision of $P$. freudenreichii into subspecies, by confirming the existence of unclassifiable strains. For this, the most relevant conditions to test $P$. freudenreichii for its activity of lactose fermentation and nitrate reduction were first determined by using a set of sequenced strains for which the expected phenotype could be predicted from their genotype. In addition, the possible relationships between the subspecies and the production of some important aroma compounds were investigated.

\section{Materials and methods}

\subsection{Strains and culture conditions}

A total of 28 strains of $P$. freudenreichii were used, including 10 previously sequenced (Loux et al. 2015) strains provided by the International Centre for Microbial Resources collection of bacteria (CIRM-BIA, UMR1253, INRA Rennes, France) and 18 Brazilian strains (Table 1). The genomic data of sequenced strains were analysed using the AGMIAL platform (Bryson et al. 2006). The Brazilian strains were isolated from dairy farms situated in Minas Gerais (Freitas et al. 2015). Before phenotypical characterization, the strains were reactivated from frozen $\left(-80^{\circ} \mathrm{C}\right)$ glycerol (Grosseron, SaintHerblain, France) stocks and grown in yeast extract lactate (YEL) medium (Malik et al. 1968) incubated at $30^{\circ} \mathrm{C}$ for $48 \mathrm{~h}$.

\subsection{Lactose fermentation and nitrate reduction}

Two concentrations of lactose and potassium nitrate were tested for a pool of strains in preliminary tests.

Lactose fermentation was tested in a modified API 50CH medium containing the following: lactose 5 or 20 g.L $\mathrm{L}^{-1}$ (Panreac, Lyon, France), tryptone 10 g.L ${ }^{-1}$, yeast extract 5 g.L ${ }^{-1}, \mathrm{~K}_{2} \mathrm{HPO}_{4}, 0.25$ g.L ${ }^{-1}, \mathrm{MnSO}_{4} 0.05$ g.L $\mathrm{L}^{-1}$, and bromocresol purple 0.17 g. $\mathrm{L}^{-1}$. The medium was inoculated using $1 \%(v / v)$ of 48 -h cultures in YEL, and incubated at $30{ }^{\circ} \mathrm{C}$ under anaerobiosis (using the Anaerocult A system, Merck, Darmstadt, Germany). The production of acid from lactose was determined from the colour change of bromocresol purple from purple to yellow after 2, 5, and 7 days of incubation.

Nitrate reductase activity was detected by means of the Griess reagent (Biomérieux, Marcy l'Etoile, France) after incubation of cultures at $30{ }^{\circ} \mathrm{C}$ under microaerophilic conditions (air atmosphere without agitation) in a broth containing potassium nitrate, 0.5 or 1.5 g.L L $^{-1}$ (VWR International, Fontenay-sous-Bois, France), tryptone (Biokar Diagnostics, Allone, France) 10 g.L ${ }^{-1}$, yeast extract (Biokar Diagnostics) 5 g.L L $^{-1}$, and glucose (Grosseron, Saint-Herblain, France) 1 g.L ${ }^{-1}$, according to Dalmasso et al. (2011). The results of the tests were read after 2 and 5 days of incubation.

All the tests were carried out in triplicate. 


\subsection{Analysis of aroma compounds}

To test strains for their ability to produce aroma compounds, cultures were grown in YEL supplemented by ethanol (final concentration $2 \mathrm{mM}$, Sigma-Aldrich, St. Quentin Fallavier, France) to promote the formation of ethyl esters (Yee et al. 2014). Volatile compounds were extracted, analysed, and identified by headspacegas chromatography-mass spectrometry (HS-GC-MS), using a TurboMatrix HS-40 trap as a headspace (HS) sampler, a Clarus 680 gas chromatograph coupled to Clarus 600T quadrupole mass spectrometer (Perkin Elmer, Courtaboeuf, France), as previously described (Pogacic et al. 2015). Briefly, samples of $2.5 \mathrm{~g}$ culture were placed in vials, they were warmed for $15 \mathrm{~min}$ at $65{ }^{\circ} \mathrm{C}$, and the volatiles were extracted at a pressure at $207 \mathrm{kPa}$ maintained in vial for 1 min with the carrier gas (helium), before being adsorbed on a Tenax ${ }^{\mathrm{TM}}$ trap at $35{ }^{\circ} \mathrm{C}$. The trap load was repeated twice for each vial trap. Volatiles were then separated on an Elite 5MS capillary column $(60 \mathrm{~m} \times 0.25 \mathrm{~mm} \times 1 \mu \mathrm{m}$; Perkin Elmer $)$, with helium as the mobile phase. The initial temperature of the oven was $35^{\circ} \mathrm{C}$, maintained for $5 \mathrm{~min}$. The oven temperature was then increased performed up to $140^{\circ} \mathrm{C}$ at a rate of $7{ }^{\circ} \mathrm{C} \cdot \mathrm{min}^{-1}$ and then up to $280^{\circ} \mathrm{C}$ at $13{ }^{\circ} \mathrm{C} \cdot \mathrm{min}^{-1}$. The mass spectrometer was operated in the scan mode, within a mass range of $m / z 25-300$, scan time $0.3 \mathrm{~s}$, and interscan delay $0.03 \mathrm{~s}$. Ionization was done by electronic impact at $70 \mathrm{eV}$. GC-MS data were processed as previously described (Pogacic et al. 2015), using the open source XCMS package implemented with the R statistical language (Smith et al., 2006), which converts the raw data to time- and mass-aligned chromatographic peaks areas. Volatile compounds were identified thanks to the mass spectral data Library NIST and to their retention index.

\subsection{Statistical analysis}

An analysis of variance (ANOVA) was performed on the abundance of selected aroma compounds from triplicate cultures, using $\mathrm{R}$ statistical software, to determine if they significantly depend on the strains and on lac/nit phenotypes. Means were compared using the least significant difference (LSD) test. A principal component analysis (PCA) was performed on preprocessed, $\log 10[x]$-transformed and Pareto scaled data, using the package FactomineR of the software R.

\section{Results}

\subsection{Lactose fermentation and nitrate reduction}

Preliminary tests were performed on a pool of 15 strains to determine the effect of varying the concentrations of lactose and potassium nitrate on the results of the tests (data not shown). The results related to lactose fermentation were the same regardless of lactose concentrations. For nitrate reduction, one strain, $P$. freudenreichii $\mathrm{B} 66$, was detected positive at $0.5 \mathrm{~g} . \mathrm{L}^{-1}$ potassium nitrate, but negative at 1.5 g.L. $\mathrm{L}^{-1}$. Therefore, the concentrations of 5 g.L $\mathrm{L}^{-1}$ lactose and 0.5 g.L $\mathrm{L}^{-1}$ nitrate were selected for further tests. 
Table 1 summarizes the results of phenotype obtained for all the strains and the genotype determined after Loux et al. (2015) for the 10 sequenced strains. The results of lactose fermentation test were negative for the two strains that did not possess a complete lactose genomic island (CIRM-BIA121 and CIRM-BIA514) and positive for the eight strains which possess a lactose genomic island, as expected. The change in colour of bromocresol purple from purple to yellow (i.e. positive test) was clear only after 5 days of incubation, or even 7 days for CIRM-BIA516 and CIRM-BIA 135, and not after 2 days. For nitrate reductase, the five strains lacking a complete nitrate (nar) genomic island were effectively found negative regardless of the incubation time. The five strains that possess the nar genomic island showed a positive phenotype, after 2 days (three strains) or only 5 days of incubation (two strains, CIRM-BIA122 and CIRM-BIA513) (Table 1).

Out of the 18 Brazilian strains, nine showed the ability to ferment lactose, with five strains positive at 2 days and 4 strains only at 5 days. Seven strains were positive for nitrate reductase, with 3 and 4 strains detected positive after 2 and 5 days of incubation, respectively.

\subsection{Production of aroma compounds}

Forty-two volatile compounds were detected in the headspace of the 28 cultures analysed (data not shown). Fifteen aroma compounds were selected among all the volatiles, based on several criteria: they are considered as important in the flavour of Swiss cheese, they result from different metabolic pathways, and they varied very significantly $(P<0.001)$ among strains (Table 2$)$. They include compounds of diverse chemical nature (alcohols, esters, carbonyl compounds, aldehydes, acids, and sulphurcontaining compounds).

The results show that the abundance of 10 of the 15 selected aroma compounds varied significantly between the four lac/nit phenotypes (freudenreichii, shermanii, $\mathrm{lac}^{+} /$nit $^{+}$and $\mathrm{lac}^{-} /$nit $^{-}$) (Table 2). However, the variations between strains within lac/ nit phenotypes were far greater that between lac/nit phenotypes, as illustrated for some compounds in Fig. 1. For example, the abundance of 2-methylbutanoic acid was 1.7fold higher in $\mathrm{lac}^{+} / \mathrm{nit}^{+}(\mathrm{P}+)$ and shermanii (S), on average, compared to freudenreichii (F) and $\mathrm{lac}^{-} /$nit $^{-}(\mathrm{P}-)$, but it showed still greater differences within the shermanii phenotype (up to 4.5-fold higher). For example, within shermanii strains, CIRMBIA118 produced less 2-methylbutanoic acid than all other strains, whereas B78 was among the highest producing strains (Fig. 1). Similarly, the abundance of propyl propanoate was six-fold higher for strains of phenotype $\mathrm{P}+$, on average, compared to $\mathrm{F}$ and $\mathrm{P}-$. However, within the phenotype $\mathrm{P}+$, the strains showed up to 20 -fold differences for the production of this compound (B82 and CIRM-BIA513 with the highest and lowest production, respectively, Fig. 1). Other compounds, such as diacetyl, ethyl propanoate, and dimethyl disulphide, did not show any significant differences between lac/nit phenotypes (Table 2, Fig. 1). The abundance of the 15 aroma compounds was subjected to PCA to visualize the proximity of strains (Fig. 2). A cumulative variation of $66 \%$ was explained by the first two principal components. PC1-PC2 plot mainly differentiated the cultures on the basis of their content in esters, branched-chain compounds, and carbonyl compounds. The four phenotypes freudenreichii, shermanii, $\mathrm{lac}^{+} /$nit $^{+}$, and $\mathrm{lac}^{-} /$nit $^{-}$appeared widespread on the map, 


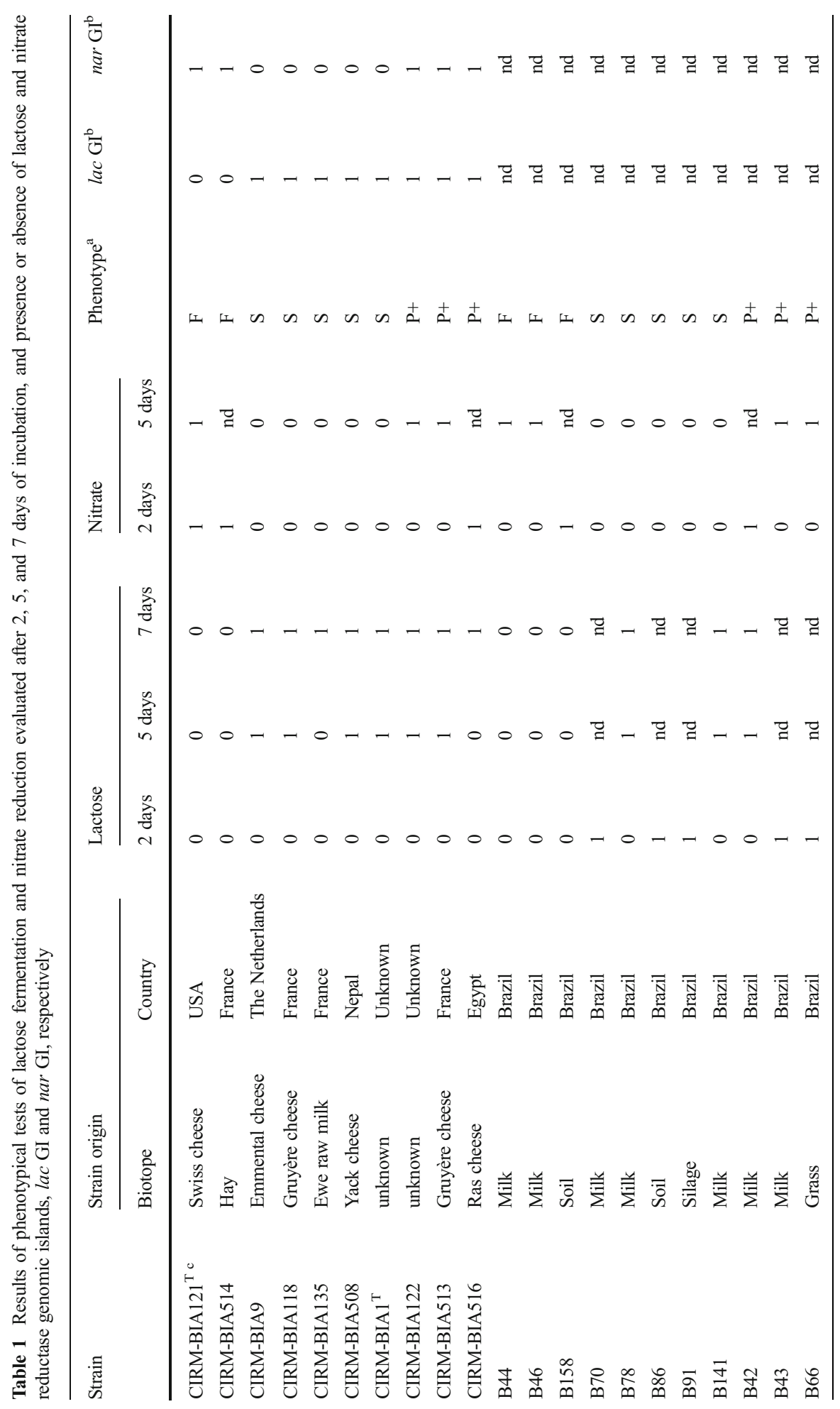

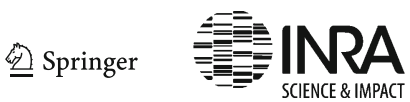




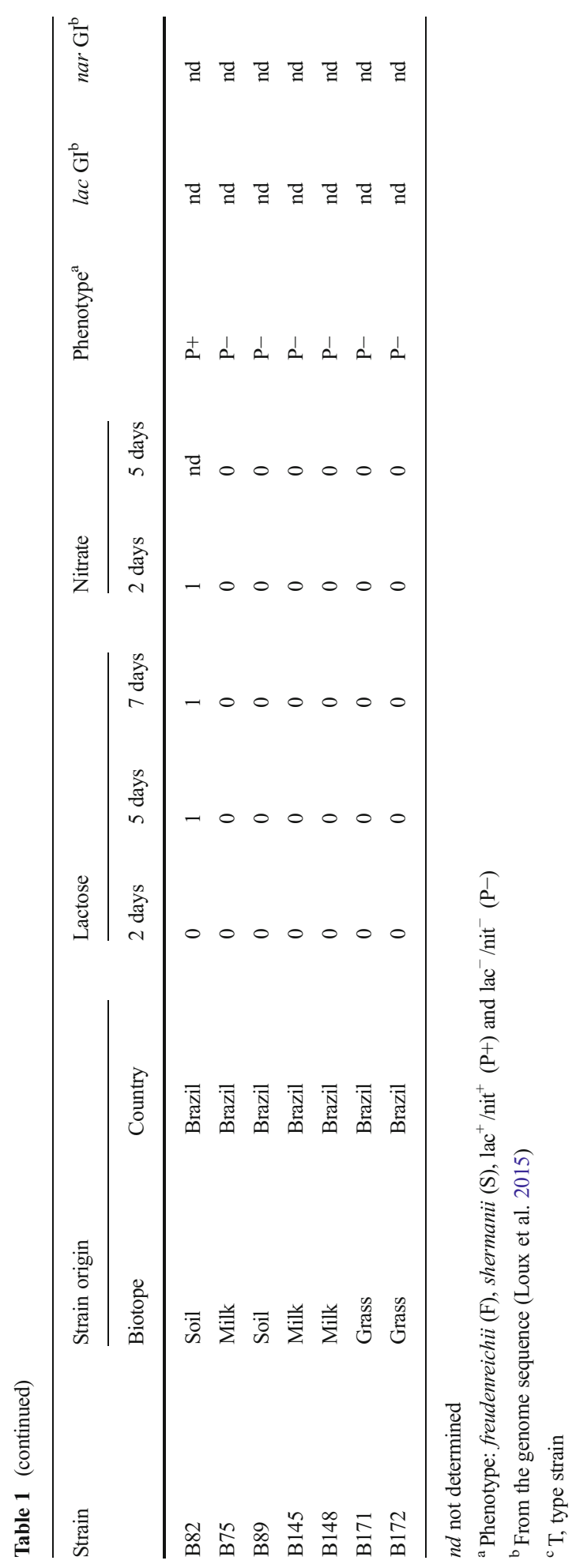


Table 2 Selected volatile aroma compounds identified in cultures of Propionibacterium freudenreichii and $P$ value of the ANOVA as function of the lac/nit phenotype and of the strain

\begin{tabular}{llllll}
\hline RI & Compounds (trivial name) & Ion $(m / z)^{\mathrm{a}}$ & Identification ${ }^{\mathrm{b}}$ & $\begin{array}{l}P \text { value, lac/nit } \\
\text { phenotype }^{\text {cd }}\end{array}$ & $\begin{array}{l}P \text { value, } \\
\text { strain }^{\mathrm{d}}\end{array}$ \\
\hline 554 & 1-Propanol & 59 & RI,D & $* * *$ & $* * *$ \\
589 & 2,3-Butanedione (diacetyl) & 86 & S,RI,D & 0.054 & $* * *$ \\
659 & 3-Methylbutanal & 58 & S,RI,D & $* * *$ & $* * *$ \\
666 & 2-Methylbutanal & 57 & S,RI,D & 0.083 & $* * *$ \\
666 & 1-Butanol & 56 & RI,D & 0.319 & $* * *$ \\
695 & 2,3-Pentanedione & 100 & RI,D & $* * *$ & $* * *$ \\
711 & Ethyl propanoate & 102 & S,RI,D & 0.697 & $* * *$ \\
712 & 2-Butanone-3-hydroxy (acetoin) & 88 & S,RI,D & $*$ & $* * *$ \\
739 & 3-Methylbutanol & 55 & S,RI,D & $* * *$ & $* * *$ \\
745 & 2-Methylbutanol & 57 & S,RI,D & $*$ & $* * *$ \\
752 & Dimethyl disulphide & 94 & S,RI,D & 0.370 & $* * *$ \\
809 & Propyl propanoate & 75 & RI,D & $* * *$ & $* * *$ \\
845 & 2-Methylbutanoic acid & 87 & RI,D & $* * *$ & $* *$ \\
872 & 3-Methylbutyl acetate & 87 & RI,D & $* *$ & $* * *$ \\
910 & Butyl propanoate & 57 & RI,D & $* *$ & $* * *$ \\
\hline
\end{tabular}

$R I$ Kovats retention index

${ }^{a}$ Ion used for quantification

${ }^{\mathrm{b}}$ Compounds identified on the basis of $\mathrm{S}$, retention time and mass spectrum from $\mathrm{S}$, standard; RI, retention index; D, mass spectral data Library NIST

${ }^{\mathrm{c}}$ lac/nit phenotypes: freudenreichii, shermanii, $\mathrm{lac}^{+} / \mathrm{nit}^{+}$, and $\mathrm{lac}^{-} / \mathrm{nit}^{-}$

${ }^{\mathrm{d}} P$ value of ANOVA: $* * * P<0.001,{ }^{* *} P<0.01, * P<0.05$

showing that the groups are not related to the ability of producing any of the aroma compounds analysed.

\section{Discussion}

This study was undertaken to re-examine the relevance of the subdivision of $P$. freudenreichii into two subspecies, which is currently based on two criteria, lactose fermentation, and nitrate reduction, and to investigate the significance of this subdivision to choose a strain as a culture for cheese manufacture.

Phenotypic methods are essential in the taxonomic characterization of microorganisms, but they are time-consuming and the results sometimes ambiguous. To give consistent results, phenotypic characterization should be applied by using strictly defined protocols adapted for each species, which are rarely available. In this study, we defined ad hoc conditions to determine lactose fermentation and nitrate reduction in $P$. freudenreichii by using 10 sequenced strains characterized for the presence or the absence of the corresponding genomic islands. The type strains of the two $P$. freudenreichii subspecies were also included. Our results show that a too short 

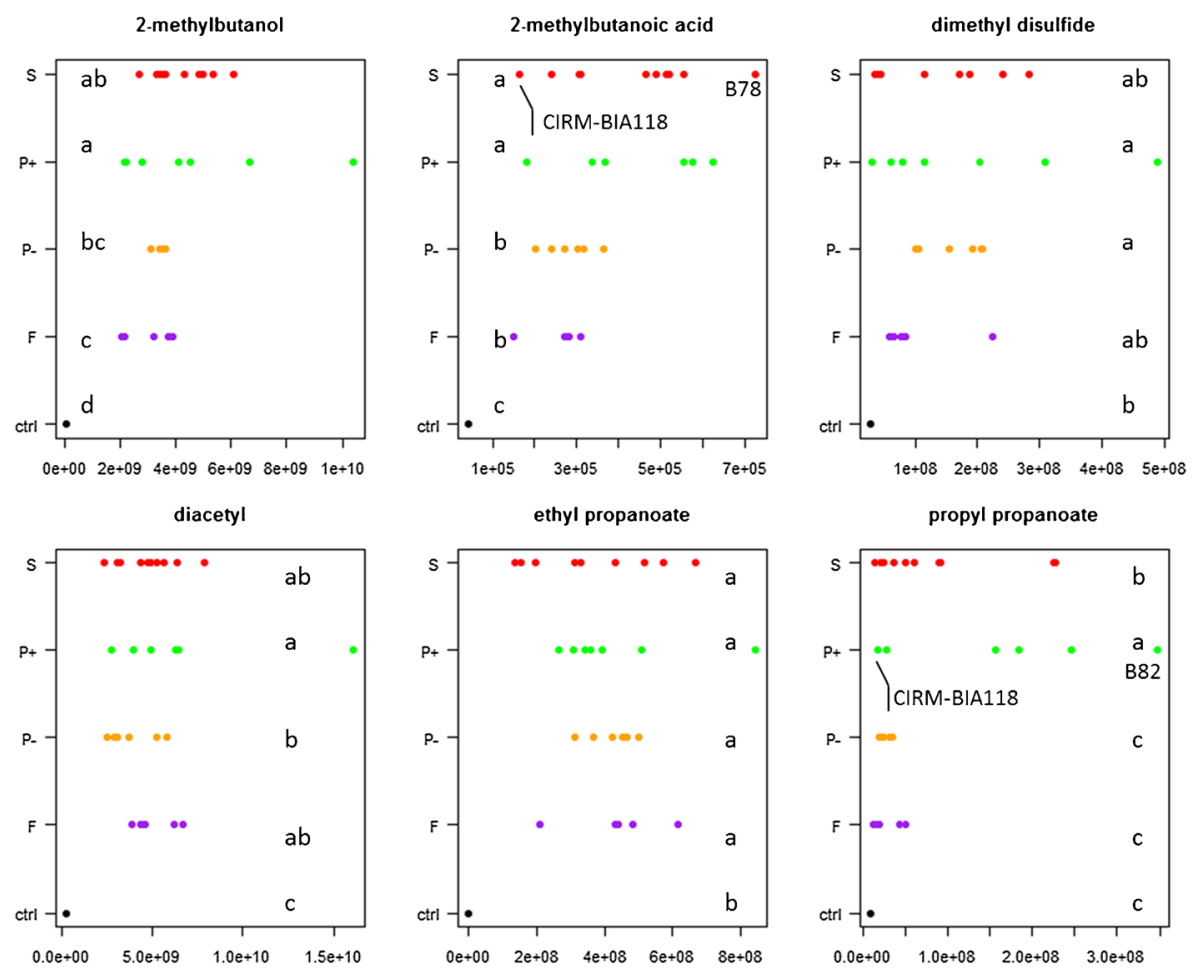

Fig. 1 Abundance of six aroma compounds, expressed as area of a specific ion $(\mathrm{m} / \mathrm{z})$, in arbitrary units, in the cultures of 28 P. freudenreichii strains of four lac/nit phenotypes (freudenreichii, shermanii, lac ${ }^{+} /$nit $^{+}$and lac $^{-}$) nit $^{-}$), abbreviated as $F, S, P+$ and $P$-, respectively). ctrl, control non-inoculated medium incubated under the same conditions; $a-d$, the production within phenotype with different letters differ significantly $(P<0.05)$

incubation time can lead to false nit ${ }^{-}$results. Swart et al. (1998) showed that Propionibacterium acidipropionici and P. freudenreichii subsp. freudenreichii were only able to reduce measurable amounts of nitrate after $70 \mathrm{~h}$ of incubation, and, more globally, that the environmental conditions have a marked effect on the ability of propionibacteria strain to reduce nitrate. Therefore, difference in the incubation time could be responsible for the discrepancies observed between this study and previous reports for the same strains. Hence, three strains previously classified as P. freudenreichii subsp. shermanii $\left(\mathrm{lac}^{+} / \mathrm{nit}^{-}\right)$CIRM-BIA122, 513, and 516 (Dalmasso et al. 2011) were identified here as nitrate reductase positive, in agreement with the phenotype expected from their genome. These three strains were thus reclassified as $\mathrm{lac}^{+} /$nit $^{+}(\mathrm{P}+$, Table 1$)$. Half of the strains characterized as nit $^{+}$in the present study actually showed a positive response only after 5 days of incubation. Our results also showed that a high concentration of potassium nitrate $\left(1.5\right.$ g.L $\left.\mathrm{L}^{-1}\right)$ in the test medium inhibited the growth of some strains, thus causing false nit ${ }^{-}$results. These results illustrate how a lack of standardization of the experimental conditions can impact the results and in particular induce the detection of false negative traits, and demonstrate the importance of the integration of reference strains in taxonomic studies.

Many strains characterized in the present study could not be classified according to the currently defined subspecies. They were identified in both the CIRM-BIA 


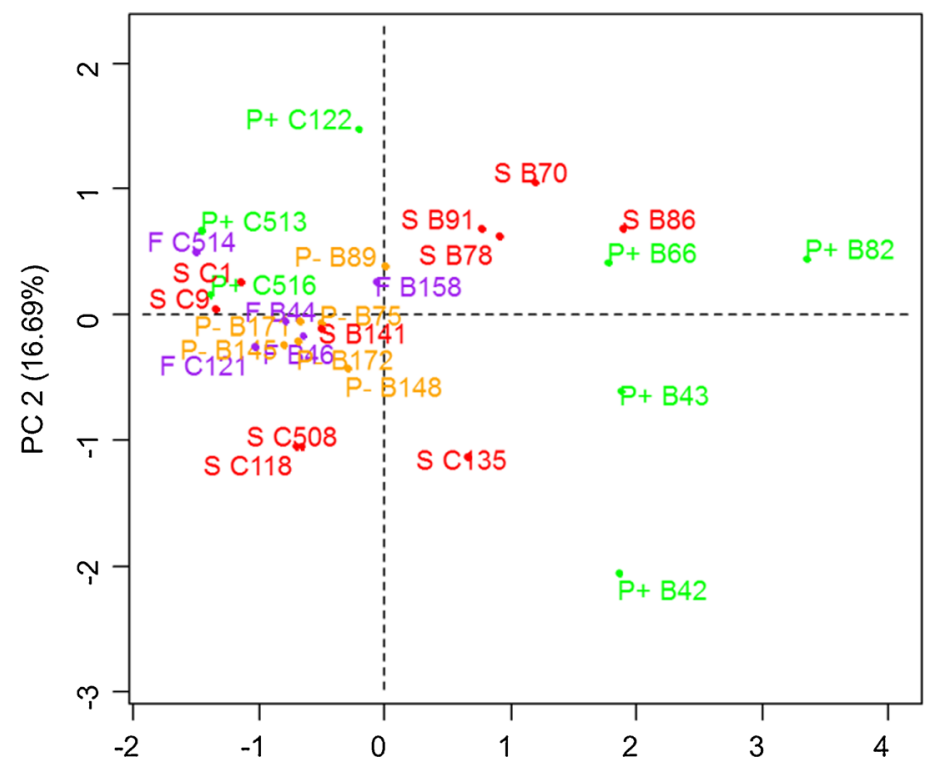

PC 1 (49.63\%)

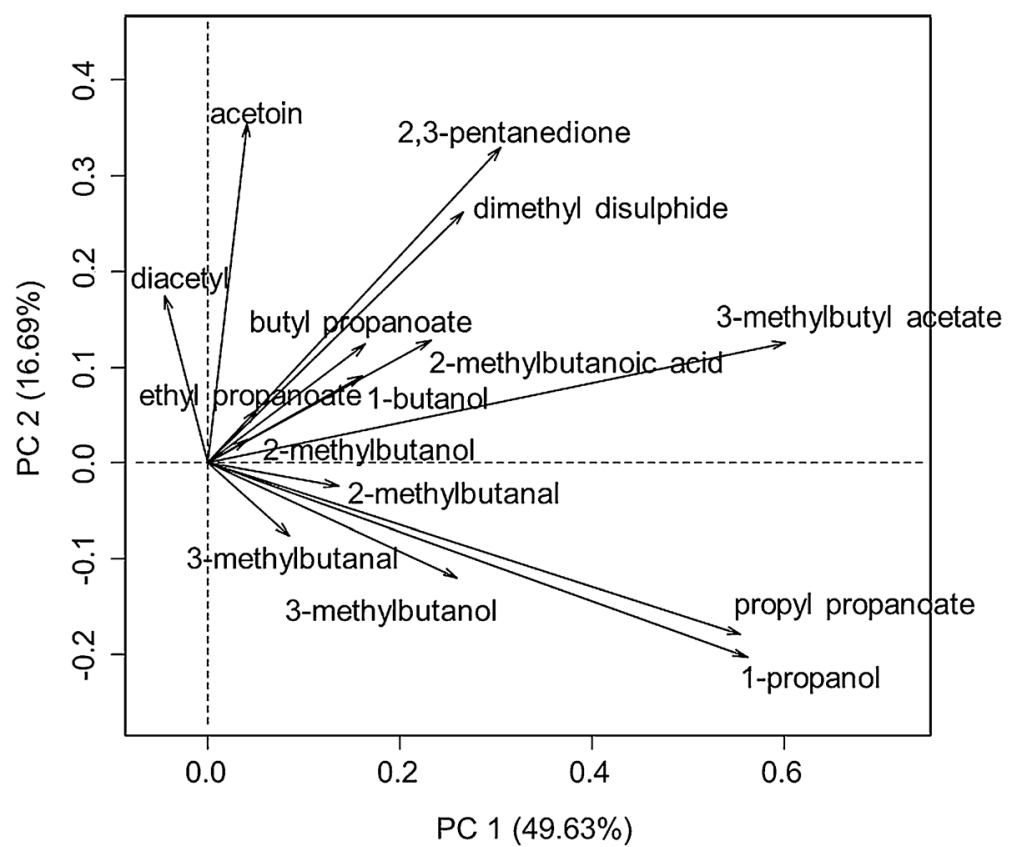

Fig. 2 Results of principal component analysis (PCA) performed on 16 aroma compounds. PCA scores and loadings for the first two principal components. Cultures are encoded using the name of the lac/nit phenotype ( $F, S, P+$ and $P-$, for freudenreichii, shermanii, $\mathrm{lac}^{+} /$nit $^{+}$and $\mathrm{lac}^{-} /$nit $^{-}$, respectively), followed by the name of strains (CIRM-BIA abbreviated as $C$ ) 
collection and the pool of Brazilian wild strains. This result is in agreement with previous reports that occasionally mentioned the existence of strains exhibiting the phenotypes $\mathrm{lac}^{+} / \mathrm{nit}^{+}$or lac $/ \mathrm{nit}^{-}$, in addition to the phenotypes corresponding to the two classical subspecies (Dalmasso et al. 2011, de Carvalho et al. 1994; Kaspar 1982; Moore and Holdeman 1974; Vorobjeva 1999). A subspecies called P. freudenreichii subsp. globosum, described as harbouring the phenotype $\mathrm{lac}^{+} / \mathrm{nit}^{+}$(de Carvalho et al. 1994), was not maintained in the first edition of the Bergey's Manual of Systematic Bacteriology (Cummins and Johnson 1986). Overall, 46\% of the strains characterized in the present study could not be classified in one of the two subspecies of $P$. freudenreichii, with $25 \%$ of $\mathrm{lac}^{+} /$nit $^{+}$and $21 \%$ of lac lnit $^{-}$strains. In comparison, $27 \%$ of the 113 strains characterized could not be classified into subspecies in the study of Dalmasso et al. (2011).

The four lac/nit phenotypes corresponding to the different combinations of lac/nit phenotypes were not distinguished by the production of any of the aroma compounds analysed. The aroma profile reflects the metabolic activity of the cells, since aroma compounds are synthesized via different pathways involving enzymes such as esterases and amino acid-converting enzymes (Abeijon Mukdsi et al. 2014, Thierry et al. 2002). The pathways of synthesis of these compounds are not related to the pathways of lactose fermentation or nitrate reduction, but these different phenotypic properties may nevertheless have been found correlated. The absence of relationship between the lac/ nit phenotypes and other metabolic activities could be expected from the fact that the $P$. freudenreichii subspecies definition does not reflect the ancestral relationships between strains, as strongly suggested by several recent studies. Using multilocus sequence typing (MLST) applied to a large collection of $P$. freudenreichii strains, Dalmasso et al. (2011) showed that the strains were distributed randomly over the phylogenetic tree regardless of their lac/nit phenotypes. Moreover, the data acquired on the genome sequence of $P$. freudenreichii CIRM-BIA $1^{\mathrm{T}}$ highlighted new insights on the origin of the random phenotypical variations concerning lactose fermentation and nitrate reduction in this species (Falentin et al. 2010). The lactose genomic island of this strain is surrounded by integrases and transposases, suggesting that the lac genes may have been acquired through horizontal transfer, whereas the gene corresponding to the $\beta$-subunit of nitrate reductase $($ nar $H)$ is a pseudo-gene due to a frameshift. Similar mechanisms may explain the very diverse phenotypes observed concerning the production of aroma compounds. The different strains tested did not group together, but rather exhibited a continuum in their ability to produce aroma compounds, as previously observed for 2-methylbutanoic acid (Dherbécourt et al. 2008) and also for free fatty acids coming from lipolysis (Abeijon Mukdsi et al. 2014).

\section{Conclusion}

This study confirms that a very large proportion of strains cannot be classified into the two currently defined subspecies of $P$. freudenreichii and demonstrates that the ability to ferment lactose and reduce nitrate are not related to each other in $P$. freudenreichii, thus generating four phenotypes based on these two criteria, and not two. Moreover, the results of this study show that belonging to a lac/nit phenotype cannot give any idea of the ability of a $P$. freudenreichii strain to produce any of the important aroma 
compounds analysed. The choice of a strain for a specific contribution in a given cheese technology should thus be done according to its specific technological properties. Taken together with some other recent studies, these results strongly suggest that maintaining the division of $P$. freudenreichii into two subspecies does not appear to be relevant.

Open Access This article is distributed under the terms of the Creative Commons Attribution License which permits any use, distribution, and reproduction in any medium, provided the original author(s) and the source are credited.

\section{References}

Abeijon Mukdsi MC, Falentin H, Maillard MB, Chuat V, Medina RB, Parayre S, Thierry A (2014) The secreted esterase of Propionibacterium freudenreichii has a major role in cheese lipolysis. Appl Environ Microbiol 80:751-756

Bryson K, Loux V, Bossy R, Nicolas P, Chaillou S, van de Guchte M, Penaud S, Maquin E, Hoebeke M, Bessières P, Gibrat J-F (2006) AGMIAL: implementing an annotation strategy for prokaryote genomes as a distributed system. Nucleic Acids Res 34:3533-3545

Busse HJ, Denner EBM, Lubitz W (1996) Classification and identification of bacteria: current approaches to an old problem. Overview of methods used in bacterial systematics. J Biotechnol 47:3-38

Cousin FJ, Denis DG, Mater DDG, Foligné B, Jan G (2011) Dairy propionibacteria as human probiotics: a review of recent evidence. Dairy Sci Technol 91:1-26

Cummins CS, Johnson JL (1986) In: Sneath PHA, Mair NS, Sharpe ME, Holt JG (eds) Bergey's manual of systematic bacteriology. Baltimore, Williams and Wilkins

Dalmasso M, Nicolas P, Falentin H, Valence F, Tanskanen J, Jatila H, Salusjärvi T, Thierry A (2011) Multilocus sequence typing of Propionibacterium freudenreichii. Int J Food Microbiol 145:113-120

Dasen G, Smutny J, Teuber M, Meile L (1998) Classification and identification of propionibacteria based on ribosomal RNA genes and PCR. Syst Appl Microbiol 21:251-259

de Carvalho AF, Gautier M, Grimont F (1994) Identification of dairy Propionibacterium species by rRNA gene restriction patterns. Res Microbiol 145:667-676

de Freitas R, Chuat V, Madec M-N, Nero LA, Valence F, Thierry A, Carvalho AF (2015) Biodiversity of dairy Propionibacterium isolated from dairy farms in Minas Gerais, Brazil. Int J Food Microbiol 203:70-77

Dherbécourt J, Maillard MB, Catheline D, Thierry A (2008) Production of branched-chain aroma compounds by Propionibacterium freudenreichii: links with the biosynthesis of membrane fatty acids. J Appl Microbiol 105:977-985

Falentin H, Deutsch SM, Jan G, Loux V, Thierry A, Parayre S, Maillard MB, Dherbecourt J, Cousin FJ, Jardin J, Siguier P, Couloux A, Barbe V, Vacherie B, Wincker P, Gibrat JF, Gaillardin C, Lortal S (2010) The complete genome of Propionibacterium freudenreichii CIRM-BIA1, a hardy actinobacterium with food and probiotic applications. PloS One 5, e11748

Fessler D, Casey MG, Puhan Z (1999) Identification of propionibacteria isolated from brown spots of Swiss hard and semi-hard cheeses. Lait 79:211-216

Hugenholtz J, Hunik J, Santos H, Smid E (2002) Nutraceutical production by propionibacteria. Lait 82:103112

Jan G, Lan A, Leverrier P (2007) In: Saarela M (ed) Functional dairy products. vol 2. Woodhead Publishing Ltd, Cambridge, UK.

Kaspar FH (1982) Nitrite reduction to nitrous oxide by propionibacteria: detoxication mechanism. Arch Microbiol 133:126-130

Loux V, Mariadassou M, Almeida S, Chiapello H, Hammani A, Buratti J, Gendrault A, Barbe V, Aury JM, Deutsch S-M, Parayre S, Madec M-N, Chuat V, Jan G, Azevedo V, Le Loir Y, Falentin H (2015) Mutations and genomic islands can explain the strain dependency of sugar utilization in 21 strains of Propionibacterium freudenreichii, BMC Genomics 16:296 doi:10.1186/s12864-015-1467-7.

Malik AC, Reinbold GW, Vedamuthu ER (1968) An evaluation of the taxonomy of Propionibacterium. Can J Microbiol 14:1185-1191

Moore WEC, Holdeman LV (1974). In: Bergey's manual of determinative bacteriology. Williams and Wilkins, Baltimore. 
Pogacic T, Maillard MB, Leclerc A, Herve C, Chuat V, Yee A, Valence F, Thierry A (2015) A methodological approach to screen diverse cheese-related bacteria for their ability to produce aroma compounds. Food Microbiol 46:145-153

Smith CA, Want EJ, O'Maille G, Abagyan R, Siuzdak G (2006) XCMS: processing mass spectrometry data for metabolite profiling using nonlinear peak alignment, matching, and identification. Anal Chem 78: 779-787

Swart R, Riedel K-H, Britz T (1998) Optimized standard conditions for determination of nitrate reduction in propionibacteria. Lait 79:217-226

Tharmaraj N, Shah NP (2009) Antimicrobial effects of probiotics against selected pathogenic and spoilage bacteria in cheese-based dips. Int Food Res J 16:261-27

Thierry A, Maillard MB, Yvon M (2002) Conversion of L-leucine to isovaleric acid by Propionibacterium freudenreichii TL 34 and ITGP23. Appl Environ Microbiol 68:608-615

Thierry A, Deutsch S-M, Falentin H, Dalmasso M, Cousin FJ, Jan G (2011) New insights into physiology and metabolism of Propionibacterium freudenreichii. Int J Food Microbiol 149:19-27

Tilsala-Timisjarvi A, Alatossava T (2001) Characterization of the 16 S-23 S and 23 S-5 S rRNA intergenic spacer regions of dairy propionibacteria and their identification with species-specific primers by PCR. Int J Food Microbiol 68:45-52

Vorobjeva LI (1999) In: Vorobjeva LI (ed) Propionibacteria. Boston, Kluwer Academic

Yee AL, Maillard MB, Roland N, Chuat V, Leclerc A, Pogačić T, Valence F, Thierry A (2014) Great interspecies and intraspecies diversity of dairy propionibacteria in the production of cheese aroma compounds. Int J Food Microbiol 191:60-68. doi:10.1016/j.ijfoodmicro.2014.09.001 